\title{
Fragmentation and systematics of the Pygmy Dipole Resonance in the stable $\mathrm{N}=82$ isotones
}

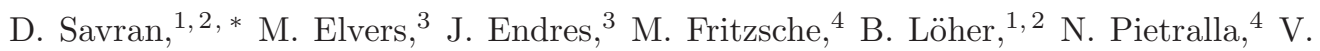 \\ Yu. Ponomarev, ${ }^{4}$ C. Romig, ${ }^{4}$ L. Schnorrenberger, ${ }^{4}$ K. Sonnabend, ${ }^{5}$ and A. Zilges ${ }^{3}$ \\ ${ }^{1}$ ExtreMe Matter Institute EMMI and Research Devision, \\ GSI Helmholtzzentrum für Schwerionenforschung GmbH, Planckstr. 1, D-64291 Darmstadt, Germany \\ ${ }^{2}$ Frankfurt Institute for Advanced Studies FIAS, Ruth-Moufang-Str. 1, D-60438 Frankfurt am Main, Germany \\ ${ }^{3}$ Institut für Kernphysik, Universität zu Köln, Zülpicher Str. 77, D-50937 Köln, Germany \\ ${ }^{4}$ Institut für Kernphysik, Technische Universität Darmstadt, \\ Schlossgartenstr. 9, D-64289 Darmstadt, Germany \\ ${ }^{5}$ Institut fur Angewandte Physik, Goethe-Universität Frankfurt, \\ Max-von-Laue-Str. 1, D-60438 Frankfurt am Main, Germany
}

(Dated: November 13, 2018)

\begin{abstract}
The low-lying electric dipole (E1) strength in the semi-magic nucleus ${ }^{136} \mathrm{Xe}$ has been measured which finalizes the systematic survey to investigate the so-called pygmy dipole resonance (PDR) in all stable even $\mathrm{N}=82$ isotones with the method of nuclear resonance fluorescence using real photons in the entrance channel. In all cases, a fragmented resonance-like structure of E1 strength is observed in the energy region $5 \mathrm{MeV}$ to $8 \mathrm{MeV}$. An analysis of the fragmentation of the strength reveals that the degree of fragmentation decreases towards the proton-deficient isotones while the total integrated strength increases indicating a dependence of the total strength on the neutron-to-proton ratio. The experimental results are compared to microscopic calculations within the quasi-particle phonon model (QPM). The calculation includes complex configurations of up to three phonons and is able to reproduce also the fragmentation of the E1 strength which allows to draw conclusions on the damping of the PDR. Calculations and experimental data are in good agreement in the degree of fragmentation and also in the integrated strength if the sensitivity limit of the experiments is taken into account.
\end{abstract}

PACS numbers: 25.20.Dc,23.20.-g,21.60.-n,21.10.Re

\section{INTRODUCTION}

In strongly coupled many-body systems, collective excitations are a common phenomenon expressing the strong interaction between the single constituents. In atomic nuclei, the classical example of collective excitations are giant resonances. The theoretical description of the damping of these collective modes within microscopic models is very difficult because of their high excitation energies and different mechanisms contributing to the damping width. The so-called Pygmy Dipole Resonance (PDR), a concentration of electric dipole (E1) strength below the well-known IsoVector Electric Giant Dipole Resonance (IVGDR) [1], has attracted considerable interest during the last few years. In contrast to the IVGDR, the PDR is an energetically low-lying mode located often (partly) below the particle thresholds. Therefore, coupling to complex configurations is the only mechanism for the resonance damping. In addition, the density of complex configurations in the energy region of the PDR is not too high allowing one to account for nearly all of them in a microscopic model. Therefore, the PDR is a challenge for theory in nuclear physics because one can expect a good description of the fragmentation of a col-

*Electronic address: d.savran@gsi.de lective mode without including any phenomenological parameters responsible for the resonance width. Also from the experimental point of view the lower energy of the PDR is advantageous since below the particle thresholds the high resolution $\gamma$-ray spectroscopy in nuclear resonance fluorescence (NRF) experiments using high-purity Germanium detectors provides the necessary energy resolution in order to study the fragmentation of the resonance. Thus, in the case of the PDR the comparison of experimental data to the results of microscopic models allows to determine whether the damping mechanism for a collective mode in many-body systems is well understood.

In the last decade, the PDR has been established as a new type of E1 excitation both experimentally as well as in theory. Many modern microscopic model calculations show an enhancement of the E1 strength at low excitation energies on top of the tail of the IVGDR, see e.g. [2, 3] and references therein. In most models the PDR exhibits the signature of an oscillation of excess neutrons against a proton-neutron core and, thus, the strength increases for more neutron-rich nuclei.

Experimental evidence for a structure of strong lowlying E1 strength in addition to the high energetic IVGDR has been found in many medium heavy to heavy nuclei. After first indications found already three decades ago [4 6] and following results with high-resolution and improved sensitivity [7-11] an experimental survey using the method of NRF has been performed in different mass 
regions in order to study the systematics of the PDR in stable nuclei [12-19]. NRF experiments are an ideal tool to investigate bound E1 strength [20]. Due to the lowmomentum transfer of real photons in the energy region of the PDR nearly exclusively $J=1$ states are populated from the ground state in even-even nuclei. Thus NRF experiments provide an excellent selectivity to the excitations of interest. Using high-purity Germanium (HPGe) detectors for the $\gamma$-ray spectroscopy of the emitted photons in the $\left(\gamma, \gamma^{\prime}\right)$ reaction an excellent energy resolution of less than $10 \mathrm{keV}$ can be achieved which allows the investigation of the fine structure and fragmentation of the E1 strength as we present in this paper. In addition, due to the well-known excitation mechanism the measured observables can be directly linked to intrinsic properties of the excited states in a model-independent way.

Currently NRF experiments using real photons are limited to stable nuclei. The E1 strength in unstable nuclei can be studied using the method of Coulomb excitations in inverse kinematics 21], which allows to extend the systematics to very neutron-rich systems. Results for neutron-rich $\mathrm{Sn}$ and $\mathrm{Sb}$ isotopes [22, 23] and ${ }^{68} \mathrm{Ni}$ 24] show an enhancement of the strength located in the $\mathrm{PDR}$ region compared to the less neutron-rich stable isotopes. This enhancement with the neutron-to-proton ratio is an important evidence for the correlation of the PDR strength to the neutron excess. Unfortunately the energy resolution in these experiments does not allow the investigation of the fine structure of the E1 strength and, thus, such investigations will only be possible for stable isotopes in the near future.

In this paper, we report on the results of a NRF experiment on ${ }^{136} \mathrm{Xe}$ which finalizes a systematic investigation of the properties of the PDR in the stable $\mathrm{N}=82$ isotones below the neutron separation energy. The results are compared in detail to calculations within the quasi-particle phonon model (QPM) with a special focus on the fragmentation of the strength. As mentioned in the first paragraph the fragmentation itself provides an important observable to compare to theory but it also has consequences on integral quantities as the total integrated strength. First results of this comparison for ${ }^{136} \mathrm{Xe}$ have recently been reported in a letter [16]. In this paper we extend the investigation of the fragmentation to all stable even $\mathrm{N}=82$ isotones and present detailed information on the experiment on ${ }^{136} \mathrm{Xe}$. In the next section the experiment and the data analysis are described, while Sec. IIIsummarizes the experimental results. Details on the QPM calculations are given in Sec. [V] In Section V] a comparison of the experimental results and the QPM calculations with respect to the fragmentation and systematics for investigated $\mathrm{N}=82$ isotones is presented.

\section{SETUP AND DATA ANALYSIS}

The experiments were performed at the Darmstadt High Intensity Photon Setup (DHIPS) using bremsstrahlung as photon source [25]. Bremsstrahlung is produced by completely stopping the electron beam of the injector module of the superconducting electron linear accelerator S-DALINAC [26] in a copper radiator where electron beam intensities of about $40 \mu \mathrm{A}$ and up to $10 \mathrm{MeV}$ are available [27]. The photon beam is collimated to a size of about $2 \mathrm{~cm}$ diameter at the target position. Three large-volume HPGe detectors, each equipped with a BGO anti-Compton shield and surrounded with heavy passive shielding, have been used for the spectroscopy of the high-energy photons emitted in the $\left(\gamma, \gamma^{\prime}\right)$ reaction. More details on the setup can be found in 25].

In the present study, two measurements have been performed. The first one using bremsstrahlung with an endpoint energy (defined by the electron energy) of $9.2 \mathrm{MeV}$ to ensure a sufficient photon flux up to the neutron separation energy of ${ }^{136} \mathrm{Xe}\left(S_{n}=8.06 \mathrm{MeV}\right)$ and another one at $8.0 \mathrm{MeV}$ just below $S_{n}$ to identify possible contributions from $(n, \gamma)$ reactions of neutrons produced by the photo-dissociation of ${ }^{136} \mathrm{Xe}$ in the $9.2 \mathrm{MeV}$ measurement.

The NRF target consisted of four high-pressure gas containers made of Titanium and filled with Xenon enriched to $99.9 \%$ in the isotope of interest. Similar targets have been used in 28] for previous NRF experiments on Xenon isotopes up to $4 \mathrm{MeV}$. In total, $2.925 \mathrm{~g}$ of ${ }^{136} \mathrm{Xe}$ was enclosed in the four containers. Further details on the high-pressure gas targets can be found in $[28$-30]. For the calibration of energy, photon flux and efficiency the ${ }^{136} \mathrm{Xe}$ was sandwiched between two ${ }^{11} \mathrm{~B}$ targets with a total mass of $0.77 \mathrm{~g}$. The combined target was smaller than the photon beam diameter and, thus, homogeneously irradiated.

An example of a measured $\gamma$-ray energy spectrum is shown in the upper part of Fig. 1 for an endpoint energy of $9.2 \mathrm{MeV}$. The spectrum has been obtained with one detector at an angle of $130^{\circ}$ with respect to the incoming beam direction. Besides peaks stemming from the ${ }^{11} \mathrm{~B}\left(\gamma, \gamma^{\prime}\right)$ reaction and a few background lines all peaks correspond to transitions in ${ }^{136} \mathrm{Xe}$.

In NRF experiments, the measured area $A_{i}$ of a peak corresponding to the elastic scattering channel of an excited state (i.e. decay back to the ground state) after photo-excitation from the ground state is related to the energy-integrated cross section $I_{i, 0}$ by

$$
A_{i} \propto I_{i, 0} \cdot W_{i}(\theta, \phi) \cdot N_{\gamma}\left(E_{i}\right) \cdot \epsilon\left(E_{\gamma}\right)
$$

with $W_{i}(\theta, \phi)$ denoting the angular distribution of the emitted photons, $N_{\gamma}\left(E_{i}\right)$ the incident photon intensity and $\epsilon\left(E_{\gamma}\right)$ the absolute photo-peak efficiency of the corresponding detector, respectively. The angular distribution function $W_{i}(\theta, \phi)$ depends exclusively on the multipole decomposition of the $\gamma$-ray transitions between the involved states and can thus be used to determine the multipolarities of the transitions and the spin quantum numbers of the excited states.

For an absolute determination of the integrated cross section $I_{i, 0}$, the product of photon intensity $N_{\gamma}\left(E_{i}\right)$ and photo-peak efficiency $\epsilon\left(E_{\gamma}\right)$ has to be calibrated. For 


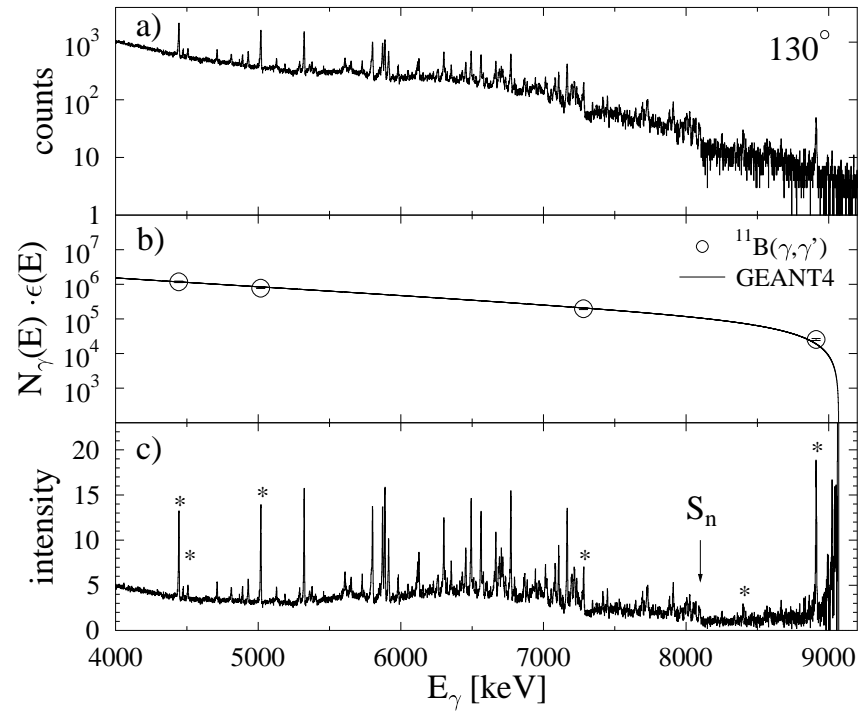

FIG. 1: a) Measured NRF spectrum for one detector at $130^{\circ}$ with respect to the incoming photon beam at an endpoint energy of $9.2 \mathrm{MeV}$. b) Determined product of photon flux $N_{\gamma}(E)$ and photopeak efficiency $\epsilon(E)$. The simulated shape has been normalized using the well-known transition in the ${ }^{11} \mathrm{~B}\left(\gamma, \gamma^{\prime}\right)$ reaction. c) Measured spectrum normalized to $N_{\gamma}(E) \cdot \epsilon(E)$. Peak areas in this intensity spectrum are directly proportional to integrated cross sections. Peaks corresponding to transitions in the normalization standard ${ }^{11} \mathrm{~B}$ are marked with an asterisk $(*)$.

transitions back to the ground state (i.e. $E_{i}=E_{\gamma}$ ), this product can be directly determined for the energies of the excited states of ${ }^{11} \mathrm{~B}$ since their NRF cross sections are known with good accuracy [31]. In order to interpolate between these energies, simulations of the bremsstrahlung spectrum and of the photopeak efficiency are performed using GEANT4 [32, 33]. It has been shown, that GEANT4 reproduces the energy dependence of our bremsstrahlung beam for the present energy region with good accuracy [25]. Finally, the product of the simulated photon spectrum and efficiency is fitted to the experimental values at the ${ }^{11} \mathrm{~B}$ energies in order to yield an absolute calibration for the entire energy region. The middle part of Fig. 1 1 shows the result of this procedure.

Since photon intensity and detection efficiency are decreasing strongly for higher photon energies the decrease in measured spectrum towards the neutron separation energy might be due to this reason. In order to correct for this effect, the measured spectrum is divided by the determined energy dependence of $N_{\gamma}\left(E_{i}\right) \cdot \epsilon\left(E_{i}\right)$. The resulting intensity spectrum is shown in the bottom part of Fig. 1. Clearly, the spectrum is dominated by the strong transitions in the energy region of about 5.5 to $7.5 \mathrm{MeV}$.

As mentioned above the spin of the excited states can be determined by measuring the angular distribution of

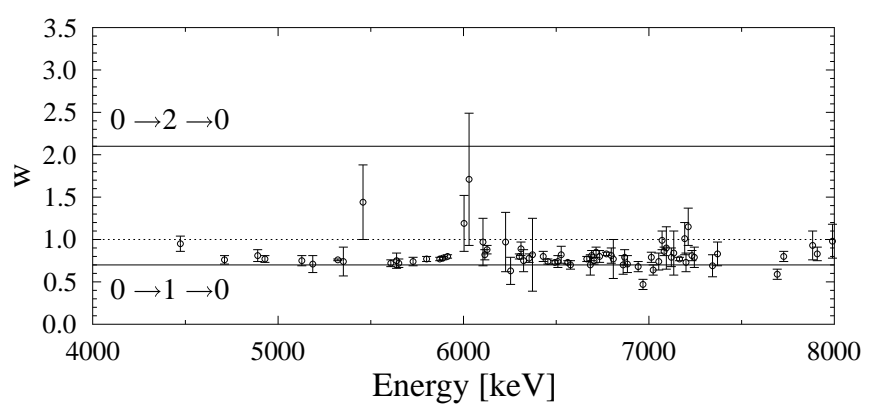

FIG. 2: Measured angular distributions for all observed transitions in ${ }^{136} \mathrm{Xe}$ in the energy region $4 \mathrm{MeV}$ to $8 \mathrm{MeV}$. Except for few weak states $J=1$ can be assigned in all cases.

the emitted photons. Figure 2 shows the ratios

$$
W=\frac{A_{i}\left(90^{\circ}\right)}{A_{i}\left(130^{\circ}\right)} \cdot \frac{\epsilon_{130^{\circ}}\left(E_{i}\right)}{\epsilon_{90^{\circ}}\left(E_{i}\right)}
$$

of the measured peak areas $A_{i}\left(90^{\circ}\right)$ and $A_{i}\left(130^{\circ}\right)$ above 3.5 MeV (corrected for the individual efficiencies) of the detectors at $90^{\circ}$ and $130^{\circ}$, respectively. The expectation values for transitions back to the ground state, i.e. the spin sequences $0_{1}^{+} \rightarrow 1 \rightarrow 0_{1}^{+}$and $0_{1}^{+} \rightarrow 2 \rightarrow 0_{1}^{+}$, are indicated by horizontal lines. Except for a few very weak transitions dipole character and thus $J=1$ for the excited states can be assigned unambiguously.

No information on the parities of the excited states could be obtained from the present data. However, investigations in the neighboring nuclei ${ }^{138} \mathrm{Ba}[17,34]$ and ${ }^{140} \mathrm{Ce}$ [35] have shown that the by far dominant part of the dipole strength has E1 character. Thus the assumption of E1 character in the case of ${ }^{136} \mathrm{Xe}$ seems to be very likely and only a small M1 contribution can be expected.

An advantage of the NRF method is the fact that transition widths can be extracted from measured cross sections in a model-independent way [20]. The connection to the integrated cross section $I_{i, 0}$ is given by

$$
I_{i, 0}=\pi^{2}\left(\frac{\hbar c}{E_{i}}\right)^{2} \cdot g \cdot \frac{\Gamma_{0}^{2}}{\Gamma}
$$

with $\Gamma_{0}$ and $\Gamma$ being the ground state and total decay width, respectively. The spin factor $g=\left(2 J_{i}+1\right) /\left(2 J_{0}+1\right)$ is given by the spin of groundstate and the excited state the latter being determined by the measured value of $W$ (compare Eq. 21). Equation (3) enables to determine the absolute ground-state decaywidth $\Gamma_{0}$ from the measured integrated cross section if the branching ratio $\Gamma_{0} / \Gamma$ is known. In the present study, no transitions corresponding to inelastic decay channels of the identified $J=1$ states could be observed. Thus, $\Gamma_{0} / \Gamma=1$ is assumed in all cases. Since many weak (and thus unobserved) branchings might add up one should keep in mind that $\Gamma_{0} / \Gamma$ might be somewhat smaller and the extracted values for $\Gamma_{0}$ represent a lower limit. However, in [17] it has been shown for ${ }^{138} \mathrm{Ba}$ that the averaged 
contribution of inelastic decays to the photo absorption cross section is small for excitation energies below $7 \mathrm{MeV}$ and becomes significant only at higher energies. Therefore, $\Gamma_{0} / \Gamma$ seems to be close to unity and the above made assumption is reasonable.

Using the determined ground state decay widths the $B(E 1) \uparrow$ transition strength can be calculated for each individual state:

$$
\frac{B(E 1) \uparrow}{\mathrm{e}^{2} \mathrm{fm}^{2}}=9.554 \cdot 10^{-4} \cdot g \cdot \frac{\Gamma_{0}}{\mathrm{meV}} \cdot\left(\frac{\mathrm{MeV}}{E_{i}}\right)^{3} .
$$

In the following chapter the results of the experiment on ${ }^{136} \mathrm{Xe}$ are presented and compared to the other $\mathrm{N}=82$ isotones.

\section{EXPERIMENTAL RESULTS}

Using the method described in the previous section the integrated cross section as well as the spin of 76 newly observed states up to the neutron separation energy are determined from the measured NRF spectra for ${ }^{136} \mathrm{Xe}$. Only in a few cases the angular distribution is also consistent with a $J=2$ assignment in all other cases $J=1$ can be clearly assigned (compare Fig. 2). Assuming $\Gamma_{0} / \Gamma=1$ (see discussion in the previous section) we obtain the corresponding ground-state decay-widths $\Gamma_{0}$ that are propotional to the excitation strengths $B(E 1) \uparrow$ in case of negative parity for the excited $J=1$ states. The results are summarized in Tab. I. No systematic deviations have been observed for the obtained cross sections between the measurements at the two different endpoint energies and, thus, the results for the single experiments have been combined.

In a previous NRF experiment 28] on ${ }^{136} \mathrm{Xe}$ with a bremsstrahlung endpoint energy of $4 \mathrm{MeV}$ only two states have been observed at $2869 \mathrm{keV}$ and $3626 \mathrm{keV}$ with $\mathrm{B}(\mathrm{E} 1)$ values of $0.169(23) \cdot 10^{-3} \mathrm{e}^{2} \mathrm{fm}^{2}$ and $1.52(12) \cdot 10^{-3} \mathrm{e}^{2} \mathrm{fm}^{2}$, respectively. While the lower lying state is below the sensitivity limit of the present experiment the higher lying one is observed and a $\mathrm{B}(\mathrm{E} 1)$ strength of $1.7(3) \cdot 10^{-3}$ $\mathrm{e}^{2} \mathrm{fm}^{2}$ has been determined. The good agreement of the two values excludes a strong feeding component in the present study even though the endpoint energy of the bremsstrahlung is above the neutron threshold.

Unlike in the other $\mathrm{N}=82$ isotones no clear and isolated candidate for the $\left[2^{+} \otimes 3^{-}\right]_{1^{-}}$two-phonon state is observed in ${ }^{136} \mathrm{Xe}$. Following the systematics of the twophonon states in the other $\mathrm{N}=82$ isotones [36] such a state would be expected around $4.2 \mathrm{MeV}$ with a strength in the order of $\mathrm{B}(\mathrm{E} 1) \approx 10 \cdot 10^{-3} \mathrm{e}^{2} \mathrm{fm}^{2}$. Around 4.5 $\mathrm{MeV}$ and slightly higher energies a few states are observed in ${ }^{136} \mathrm{Xe}$ that however have smaller individual $\mathrm{B}(\mathrm{E} 1)$ strengths than expected for the two-phonon state. Thus, the $\left[2^{+} \otimes 3^{-}\right]_{1}$ - strength is either fragmented into several states that share the two-phonon E1 strength or its strength is further reduced.
TABLE I: Results of the experiment on ${ }^{136}$ Xe. Given are deduced energy, angular distribution, spin, partial decay width and $B(E 1) \uparrow$ strength of the observed states. For the latter $\pi=-1$ and $\Gamma_{0} / \Gamma=1$ was used, since no branching transitions were observed.

\begin{tabular}{|c|c|c|c|c|}
\hline $\begin{array}{l}E_{x} \\
(\mathrm{keV})\end{array}$ & W & $\begin{array}{l}J^{\pi} \\
(\hbar)\end{array}$ & $\begin{array}{c}\Gamma_{0}^{2} / \Gamma \\
(\mathrm{eV})\end{array}$ & $\begin{array}{c}B(E 1) \uparrow \\
\left(10^{-3} \mathrm{e}^{2} \mathrm{fm}^{2}\right)\end{array}$ \\
\hline $3626(1)^{a}$ & $1.20(45)$ & 1,2 & $0.027(6)$ & $1.7(3)^{a}$ \\
\hline $3738(1)$ & $0.93(13)$ & 1 & $0.057(5)$ & $3.1(3)$ \\
\hline $4473(1)$ & $0.95(9)$ & 1 & $0.120(8)$ & $3.9(3)$ \\
\hline 4711(1) & $0.76(5)$ & 1 & $0.217(13)$ & $6.0(4)$ \\
\hline 4890(1) & $0.81(7)$ & 1 & $0.175(11)$ & $4.3(3)$ \\
\hline $4929(1)$ & $0.77(4)$ & 1 & $0.341(19)$ & $8.1(5)$ \\
\hline $5128(1)$ & $0.75(6)$ & 1 & $0.199(13)$ & $4.2(3)$ \\
\hline $5187(1)$ & $0.71(10)$ & 1 & $0.088(8)$ & $1.8(2)$ \\
\hline $5322(1)$ & $0.76(1)$ & 1 & $1.66(9)$ & $31.6(16)$ \\
\hline $5352(1)$ & $0.74(17)$ & 1 & $0.072(9)$ & $1.3(2)$ \\
\hline $5458(1)$ & $1.44(44)$ & 1,2 & $0.122(17)$ & $2.2(3)$ \\
\hline $5608(1)$ & $0.72(4)$ & 1 & $0.491(29)$ & $8.0(5)$ \\
\hline $5639(1)$ & $0.75(9)$ & 1 & $0.210(17)$ & $3.4(3)$ \\
\hline $5651(1)$ & $0.72(5)$ & 1 & $0.441(26)$ & $7.0(4)$ \\
\hline $5728(1)$ & $0.74(5)$ & 1 & $0.382(24)$ & $5.8(4)$ \\
\hline $5801(1)$ & $0.77(3)$ & 1 & $2.02(11)$ & $29.7(16)$ \\
\hline $5872(1)$ & $0.77(2)$ & 1 & $2.05(11)$ & $29.1(15)$ \\
\hline $5888(1)$ & $0.78(2)$ & 1 & $2.59(13)$ & $36.3(19)$ \\
\hline $5914(1)$ & $0.80(2)$ & 1 & $1.36(7)$ & $18.9(10)$ \\
\hline $6003(1)$ & $1.19(33)$ & 1,2 & $0.126(18)$ & $1.7(2)$ \\
\hline $6030(1)$ & $1.71(78)$ & 1,2 & $0.088(16)$ & $1.1(2)$ \\
\hline $6105(1)$ & $0.97(28)$ & 1 & $0.159(26)$ & $2.0(3)$ \\
\hline $6115(1)$ & $0.82(6)$ & 1 & $0.661(48)$ & $8.3(6)$ \\
\hline $6127(1)$ & $0.88(5)$ & 1 & $1.10(7)$ & $13.7(9)$ \\
\hline $6227(1)$ & $0.97(35)$ & 1 & $0.304(50)$ & $3.6(6)$ \\
\hline $6254(1)$ & $0.63(16)$ & 1 & $0.236(32)$ & $2.8(4)$ \\
\hline 6301(1) & $0.80(3)$ & 1 & $1.99(11)$ & $22.8(12)$ \\
\hline $6310(1)$ & $0.89(8)$ & 1 & $0.629(42)$ & $7.2(5)$ \\
\hline $6324(1)$ & $0.75(13)$ & 1 & $0.214(22)$ & $2.4(2)$ \\
\hline $6354(1)$ & $0.78(5)$ & 1 & $0.796(47)$ & $8.9(5)$ \\
\hline $6372(1)$ & $0.82(43)$ & 1 & $0.108(30)$ & $1.2(3)$ \\
\hline $6430(1)$ & $0.80(6)$ & 1 & $0.643(40)$ & $6.9(4)$ \\
\hline $6455(1)$ & $0.74(3)$ & 1 & $1.38(8)$ & $14.7(8)$ \\
\hline $6493(1)$ & $0.73(2)$ & 1 & $2.62(14)$ & $27.5(14)$ \\
\hline $6509(1)$ & $0.74(7)$ & 1 & $0.489(34)$ & $5.1(4)$ \\
\hline $6527(1)$ & $0.82(10)$ & 1 & $0.410(32)$ & $4.2(3)$ \\
\hline $6562(1)$ & $0.73(2)$ & 1 & $2.43(13)$ & $24.6(13)$ \\
\hline $6577(1)$ & $0.70(5)$ & 1 & $0.703(43)$ & $7.1(4)$ \\
\hline $6665(1)$ & $0.77(3)$ & 1 & $1.81(10)$ & $17.5(10)$ \\
\hline $6684(1)$ & $0.70(12)$ & 1 & $0.482(47)$ & $4.6(4)$ \\
\hline $6691(1)$ & $0.81(6)$ & 1 & $1.19(8)$ & $11.4(7)$ \\
\hline $6704(1)$ & $0.75(4)$ & 1 & $1.32(8)$ & $12.5(7)$ \\
\hline $6715(1)$ & $0.85(6)$ & 1 & $1.17(7)$ & $11.0(7)$ \\
\hline $6734(1)$ & $0.80(7)$ & 1 & $0.673(44)$ & $6.3(4)$ \\
\hline $6771(1)$ & $0.83(2)$ & 1 & $3.46(18)$ & $31.9(17)$ \\
\hline $6797(1)$ & $0.81(9)$ & 1 & $0.542(40)$ & $4.9(4)$ \\
\hline $6808(1)$ & $0.77(23)$ & 1 & $0.190(28)$ & $1.7(3)$ \\
\hline $6861(1)$ & $0.70(11)$ & 1 & $0.407(38)$ & $3.6(3)$ \\
\hline $6869(1)$ & $0.79(9)$ & 1 & $0.621(48)$ & $5.5(4)$ \\
\hline $6884(1)$ & $0.71(10)$ & 1 & $0.342(29)$ & $3.0(3)$ \\
\hline $6942(1)$ & $0.68(6)$ & 1 & $0.668(45)$ & $5.7(4)$ \\
\hline $6968(1)$ & $0.47(6)$ & 1 & $0.338(30)$ & $2.9(3)$ \\
\hline $7013(1)$ & $0.79(06)$ & 1 & $1.01(6)$ & $8.4(5)$ \\
\hline $7023(1)$ & $0.64(06)$ & 1 & $0.683(47)$ & $5.6(4)$ \\
\hline $7053(1)$ & $0.74(10)$ & 1 & $0.398(33)$ & $3.3(3)$ \\
\hline $7071(1)$ & $0.99(11)$ & 1 & $0.670(49)$ & $5.4(4)$ \\
\hline $7082(1)$ & $0.86(05)$ & 1 & $1.44(8)$ & $11.6(7)$ \\
\hline $7094(1)$ & $0.90(25)$ & 1 & $0.283(44)$ & $2.3(4)$ \\
\hline $7121(1)$ & $0.79(11)$ & 1 & $0.394(34)$ & $3.1(3)$ \\
\hline $7134(1)$ & $0.84(26)$ & 1 & $0.247(42)$ & $1.9(3)$ \\
\hline $7165(1)$ & $0.77(2)$ & 1 & $3.43(18)$ & $26.7(14)$ \\
\hline $7193(1)$ & $1.01(19)$ & 1 & $0.74(8)$ & $5.7(6)$ \\
\hline $7200(1)$ & $0.73(11)$ & 1 & $0.84(8)$ & $6.5(6)$ \\
\hline $7212(1)$ & $1.15(22)$ & 1 & $0.91(9)$ & $6.9(7)$ \\
\hline $7232(1)$ & $0.81(6)$ & 1 & $1.00(7)$ & $7.6(5)$ \\
\hline $7245(1)$ & $0.79(12)$ & 1 & $0.361(38)$ & $2.7(3)$ \\
\hline $7343(1)$ & $0.69(13)$ & 1 & $0.292(31)$ & $2.1(2)$ \\
\hline $7370(1)$ & $0.83(14)$ & 1 & $0.357(35)$ & $2.6(3)$ \\
\hline $7692(1)$ & $0.59(6)$ & 1 & $0.78(6)$ & $4.9(4)$ \\
\hline $7727(1)$ & $0.80(6)$ & 1 & $1.69(11)$ & $10.5(7)$ \\
\hline $7883(1)$ & $0.93(17)$ & 1 & $0.80(9)$ & $4.7(5)$ \\
\hline $7908(1)$ & $0.83(8)$ & 1 & $1.65(14)$ & $9.6(8)$ \\
\hline $7990(1)$ & $0.98(20)$ & 1 & $0.75(9)$ & $4.2(5)$ \\
\hline $8024(1)$ & $0.76(8)$ & 1 & $1.40(12)$ & $7.8(7)$ \\
\hline $8051(1)$ & $0.93(13)$ & 1 & $1.11(11)$ & $6.1(6)$ \\
\hline $8066(1)$ & $0.75(12)$ & 1 & $0.86(9)$ & $4.7(5)$ \\
\hline $8093(1)$ & $0.77(10)$ & 1 & $1.02(10)$ & $5.5(5)$ \\
\hline
\end{tabular}




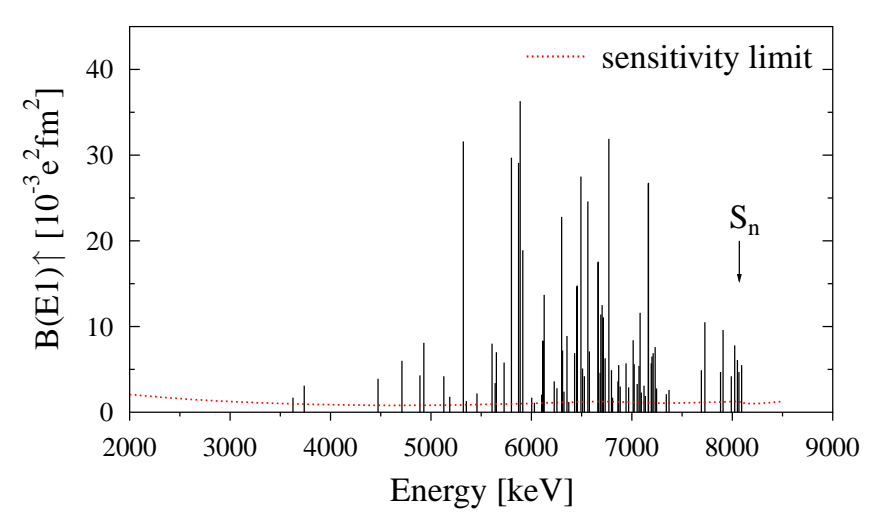

FIG. 3: (Color online) Measured E1 strength distribution in ${ }^{136} \mathrm{Xe}$ up to the neutron separation energy. The dotted line indicates the sensitivity limit of the experiment.

The measured B(E1) strength distribution for ${ }^{136}$ Xe up to $S_{n}$ is shown in the upper part of Fig. 3. The sensitivity limit of the experiment is indicated by the dotted line and has been calculated based on the continuous background present in the spectra and following the formalism given in [37] using a confidence limit of $3 \sigma$. Due to this finite sensitivity excitations with smaller $B(E 1) \uparrow$ values thus will remain unobserved.

Similar as in the other stable $\mathrm{N}=82$ isotones also in ${ }^{136} \mathrm{Xe}$ a concentration of strong $\mathrm{E} 1$ excitations in the energy region of $5 \mathrm{MeV}$ to $7 \mathrm{MeV}$ is observed. The left part of Fig. 4 shows the measured B(E1) strength distributions for all $\mathrm{N}=82$ isotones. The distribution of ${ }^{136} \mathrm{Xe}$ fits into the systematics with an increasing strength and also increasing excitation energy compared to the heavier isotones. A more detailed comparison of the $\mathrm{B}(\mathrm{E} 1)$ strength distribution in terms of total strength and also fragmentation is presented in Sec. $\nabla$

The facts that the experimental sensitivity is always limited and, in addition, branching transitions are difficult to observe consequently lead to an incomplete determination of the total E1 strength using the presented experimental technique. Recently, attempts have been made to extract also the strength in the continuum part of the experimental spectra and in parallel to account for the inelastic decay branchings in order to estimate the total photo absorption cross section [18, 19, 38]. However, in this approach one loses the model independence of the NRF method and also highly relies on very accurate simulations of background and detector response functions. Therefore, we do not follow this approach here especially since the contribution of unresolved strength in the case of the semi-magic ${ }^{136} \mathrm{Xe}$ isotope can be expected to be lower and the inelastic contribution has been shown to be rather small in the neighboring nucleus ${ }^{138} \mathrm{Ba}$ [17] in the energy region of interest. Even though the total strength extracted will then represent a lower limit, the result remains model independent which is one of the great advantages of real photon scattering experiments and still allow for a solid and unambiguous comparison to theory as it is done below.

In a comparison to model calculations this limited knowledge about the total excitation strength needs to be accounted for. This can be done by comparing only the part of the strength produced in a model calculation which is above the experimental sensitivity and, thus, can be expected to be observed in the experiment. Of course, to allow such a kind of comparison the fragmentation of the E1 strength has to be reproduced correctly in the model in order to account for the experimental sensitivity. The QPM calculations presented in the next section fulfill this requirement as we will show in Sec. V.

\section{QPM CALCULATIONS}

Excited states of even-even nuclei are treated in the quasi-particle phonon model (QPM) [39] in terms of phonons with spin and parity $\lambda^{\pi}$; the ground state is considered as a phonon vacuum. The phonons are made up of quasi-particle pairs. Their spectra and internal fermion structure is obtained by solving the QRPA equations for each multipolarity. The QRPA involves $0 p 4 h$, $2 p 2 h$, and $4 p 0 h$ terms of the residual two-body interaction. This interaction in the QPM has a simple separable form. The remaining $1 p 3 h$ and $3 p 1 h$ terms of the residual interaction are responsible for the mixing between one- and two-, two- and three-, etc. phonon configurations. Accordingly, the wave function of excited states in the QPM is written as a composition of one-, two-, etc phonon configurations. The energies of excited states and components of their wave functions are found from a diagonalization of the model Hamiltonian on the set of these wave functions.

Although the QPM wave functions have a complex form their one-phonon components play a decisive role in the excitation process of these states from the ground state by an external field (e.g., electromagnetic) described by a one-body operator. Two-phonon components are also excited from the QRPA ground state. An example is the first $1^{-}$state in spherical nuclei which has almost pure $\left[2_{1}^{+} \otimes 3_{1}^{-}\right]_{1^{-}}$nature. But in general, their transition matrix elements are much smaller compared to the ones of one-phonon components. Thus, complex (two-, three-phonon) configurations participate in the creation of the fragmentation pattern of the excitation strength but add very little to the total strength.

The formation of the fragmentation pattern is demonstrated in Fig. 5 in which the distribution of the E1 strength of the PDR in ${ }^{136} \mathrm{Xe}$ is presented. Figure $5 \mathrm{~b}$ presents the results obtained in the one-phonon approximation. One notices that the E1 strength in this energy region originates from four one-phonon states. Calculations performed with the wave function containing oneand two-phonon configurations are shown in Fig. $5 \mathrm{~b}$. The $\mathrm{B}(\mathrm{E} 1)$ value for each individual $1^{-}$state drops dramatically as compared to the results in Fig. 5 . This is due 


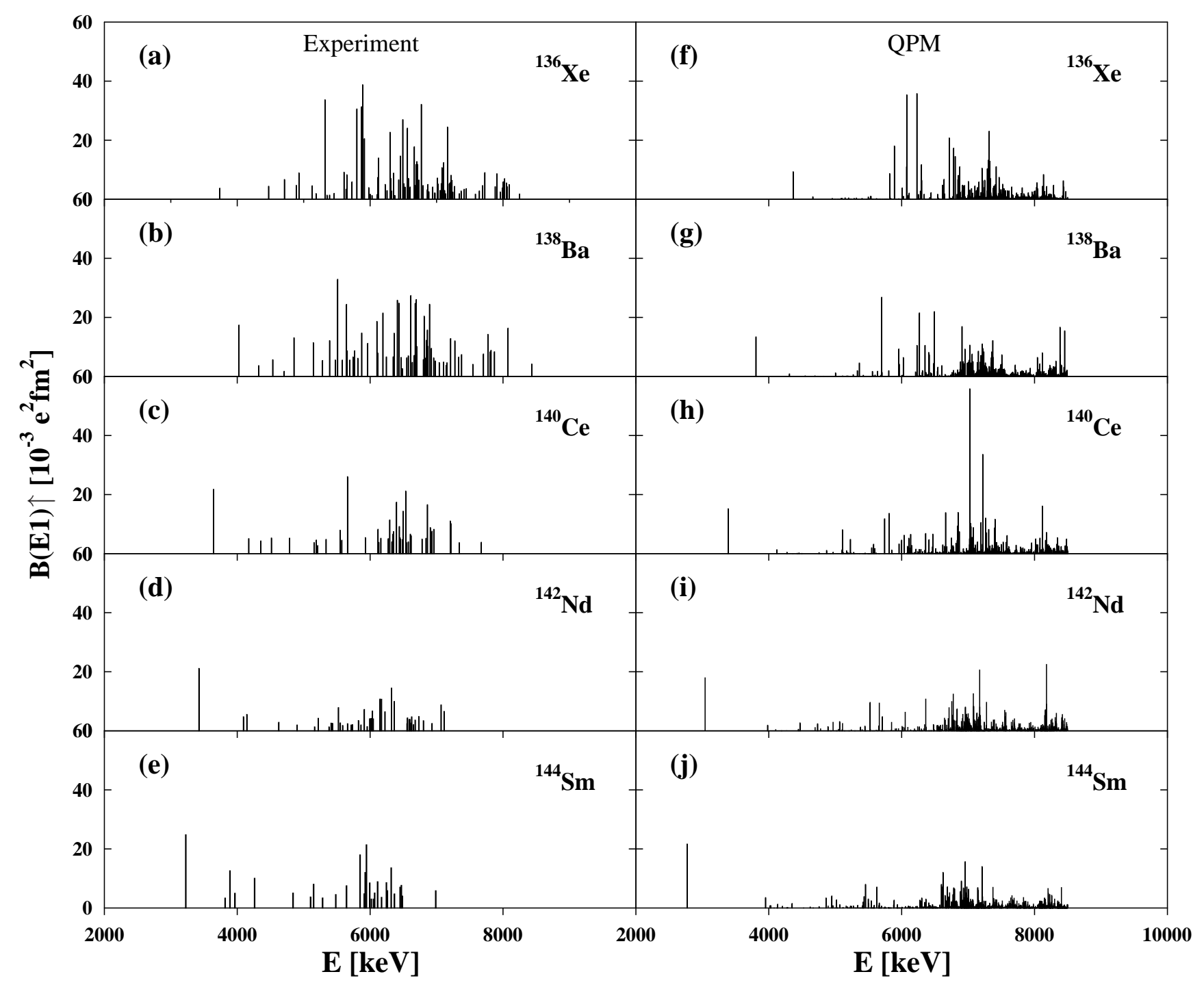

FIG. 4: Measured (left side) and calculated (right side) B(E1) strength distributions in the stable even N=82 isotones. The experimental sensitivity limits of all experiments are similar to the one shown for ${ }^{136}$ Xe in Fig. 3] Data taken partly from [15].

to the fact that the number of two-phonon configurations in this energy interval is much larger. Interaction between one- and two-phonon configurations leads to their mixing and the contribution of the one-phonon configurations (which carry E1 strength) to the wave function norm does not exceed a few percents for each state. Note also the appearance of the two-phonon $1^{-}$state discussed above at around $4.5 \mathrm{MeV}$ in this step. The fragmentation progresses further when three-phonon configurations are added (Fig. 5.). Many states with rather small B(E1) values appear especially at higher energies with rapid increase of the density of three-phonon configurations.

The QPM calculations of the fine structure of the PDR in the $\mathrm{N}=82$ isotones in this paper extend previous calculations in ${ }^{138} \mathrm{Ba}$ [40] and ${ }^{140} \mathrm{Ce}[8]$ which together with Ref. 9] were the first theoretical studies on the topic. Compared to the old calculations we have enlarged the basis of complex configurations: two- and three-phonon configurations have been built up from phonons with the multipolarity from $1^{ \pm}$up to $9^{ \pm}$with the excitation energy below $8.5 \mathrm{MeV}$. Since the density of four-phonon configurations (not included in the wave function) is still very low at these energies, our basis is almost complete. The number of complex configurations fluctuates slightly from nucleus to nucleus. On average, our basis contains about 350 two-phonon and 900 three-phonon configurations. To account for admixture of the GDR in the lowenergy region, all $1^{-}$one-phonon configurations below $20 \mathrm{MeV}$ have been included in the wave function of excited states.

The calculations in all $\mathrm{N}=82$ isotones have been performed with the same mean field which has been described by the Woods-Saxon potential with parameters taken from a global parametrization [41] and the same 


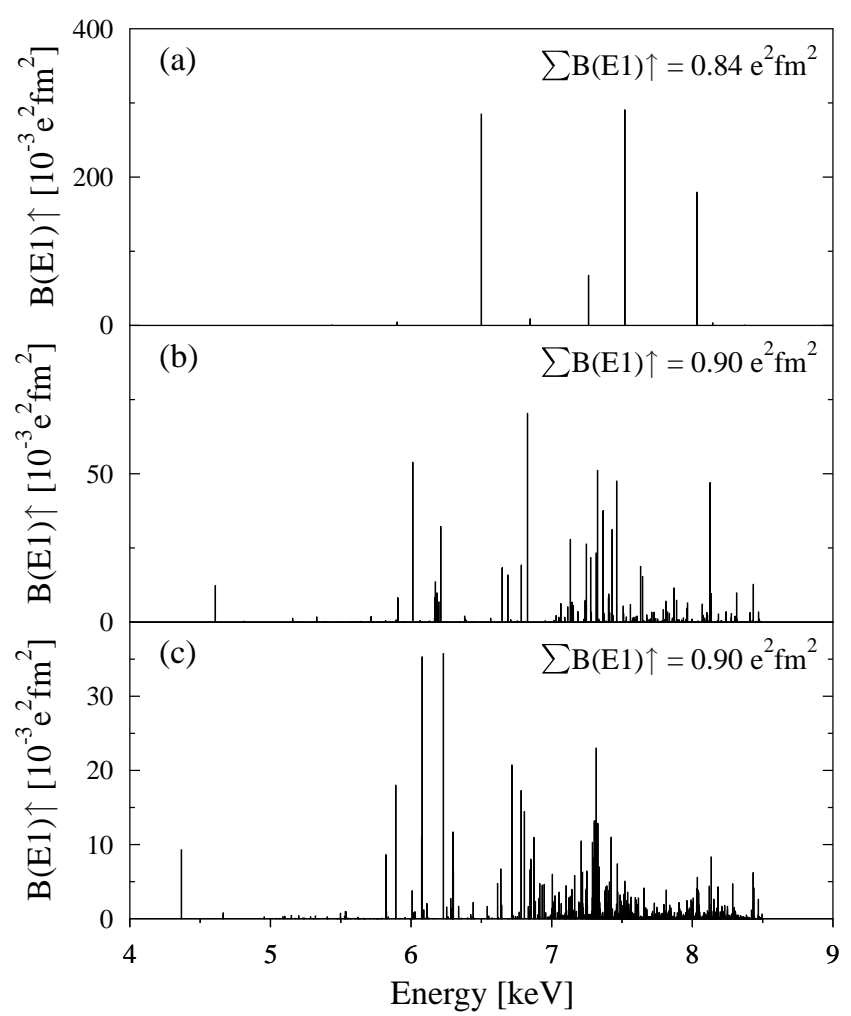

FIG. 5: The QPM calculations of the B(E1) strength distribution in ${ }^{136} \mathrm{Xe}$ performed a) in one-phonon approximation and with the wave function containing b) one- and twoand c) one-, two-, and three-phonon configurations. The summed strength is hardly influenced while the fragmentation increases rapidly.

monopole pairing strength. Single-particle energies of the mean field near the Fermi surface have been corrected to reproduce the experimentally known single-particle levels in neighboring odd-mass nuclei in the calculations with the wave function containing "[quasiparticle $\otimes \mathrm{N}$ phonon]" $(N=0,1,2,3)$ components. The strength parameters of the residual interaction have been adjusted in each particular nucleus in accordance with a standard QPM procedure (see, e.g., [42]).

Figure 6] shows calculated transition densities separately for protons an neutron for the PDR region in ${ }^{136}$ Xe. Very similar transition densities are obtained for the other $\mathrm{N}=82$ isotones and are thus not shown here.

\section{COMPARISON}

A comparison between the experimentally determined $\mathrm{B}(\mathrm{E} 1)$ strength distributions and the results of the QPM calculations for all stable even $\mathrm{N}=82$ isotones is shown in Fig. 4. Besides a shift of about $500 \mathrm{keV}$ there is in general a good agreement between experiment and QPM in the absolute scale of the strongest excitations and the shape of the distributions. In all cases a resonance-like

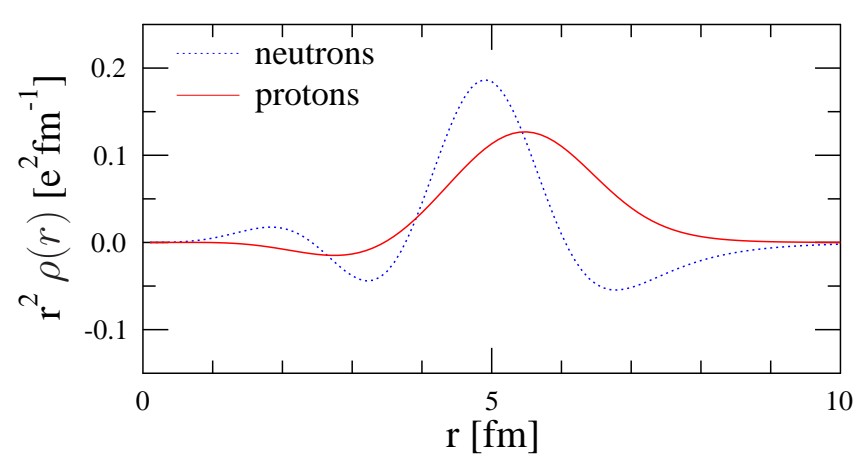

FIG. 6: (Color online) Calculated transition densities for protons (red) and neutrons (blue) in the PDR region for ${ }^{136} \mathrm{Xe}$.

structure is observed in experiment as well as in the calculation which increases in energy and strength from ${ }^{144} \mathrm{Sm}$ to ${ }^{136} \mathrm{Xe}$.

In order to compare the fragmentation of the E1 strength produced within the QPM with the experimental result the $\mathrm{B}(\mathrm{E} 1)$ strength distribution is shown in Fig. 7 as a function of the strength of the individual states. To produce Fig. 7 for each nucleus the states have been grouped according to their $\mathrm{B}(\mathrm{E} 1)$ strengths in bins of $1 \cdot 10^{-3} \mathrm{e}^{2} \mathrm{fm}^{2}$ and the summed $\sum \mathrm{B}(\mathrm{E} 1)$ strength is calculated for each bin. Figure 7 thus shows how the strength is distributed (fragmented) over the individual states. In this way, the limited experimental sensitivity is expressed by the fact that no or only little strength is observed for the group of states with very small $\left(\leq 2 \cdot 10^{-3}\right.$ $\left.\mathrm{e}^{2} \mathrm{fm}^{2}\right)$ strength. For these bins, the experiment clearly misses strength. However, for stronger states the experimental results can be expected to be complete and thus, this part needs to be compared to the QPM calculation. For the region above about $3 \cdot 10^{-3} \mathrm{e}^{2} \mathrm{fm}^{2}$ experiment and calculation show indeed very similar distributions. The QPM therefore nicely reproduces the experimental distribution, i.e. the fragmentation is described correctly within the model. This confirms that the damping mechanism of the PDR is the coupling of a collective mode with complex configurations. For the weaker excitations the QPM predicts much more strength than is observed experimentally which clearly is a sign of the limited experimental sensitivity. However, since the QPM reproduces the experimental results for stronger states, the amount of strength located in states with small B(E1) values in the QPM can be expected to be a good estimate for the missing experimental strength.

Both, experiment and QPM, show a decreasing amount of excitations with large $\mathrm{B}(\mathrm{E} 1)$ strength with increasing proton number. While in ${ }^{136}$ Xe several states with $\mathrm{B}(\mathrm{E} 1)$ values well above $20 \cdot 10^{-3} \mathrm{e}^{2} \mathrm{fm}^{2}$ exist, there is only one in ${ }^{142} \mathrm{Nd}$ or ${ }^{144} \mathrm{Sm}$. In general the strength is distributed among more and more states with small E1 excitations strengths for increasing proton number, i.e. the fragmentation increases. This is related to the fact that the en- 


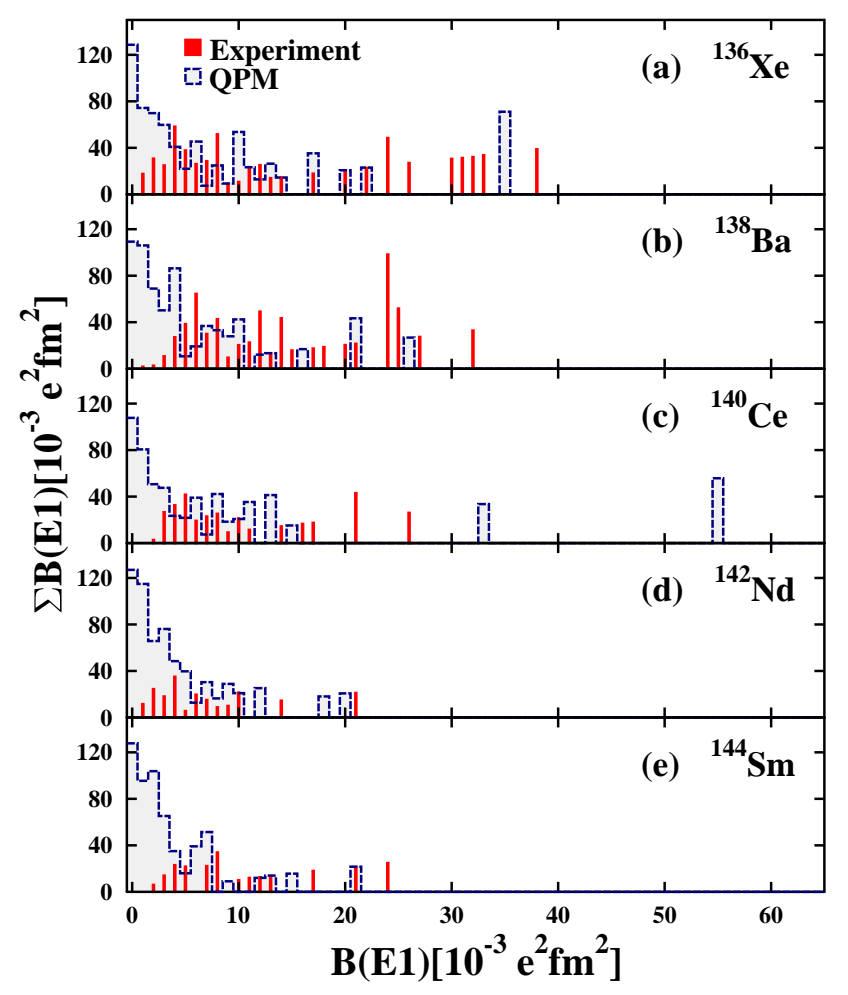

FIG. 7: (Color online) Analysis of the fragmentation in the experimentally observed distribution and within the QPM model for the $\mathrm{N}=82$ isotones. For details see text.

ergy of the $3_{1}^{-}$state systematically decreases from ${ }^{136} \mathrm{Xe}$ to ${ }^{144} \mathrm{Sm}$ while the $2_{1}^{+}$state has almost the same energy in all isotones. In other words, collectivity of the $3_{1}^{-}$state increases from light to heavy isotones making coupling matrix elements between one-phonon $1^{-}$configurations and many complex configurations stronger.

The integrated E1 strength up to $8.06 \mathrm{MeV}$ (the neutron separation energy of ${ }^{136} \mathrm{Xe}$ ) for the five investigated $\mathrm{N}=82$ isotones is shown in Fig. 8 as a function of the neutron-to-proton ratio $\mathrm{N} / \mathrm{Z}$ and summarized in Tab. II. The experimental values show a rather strong dependence on $\mathrm{N} / \mathrm{Z}$ with values of $0.220(37) \mathrm{e}^{2} \mathrm{fm}^{2}$ for ${ }^{144} \mathrm{Sm}$ and $0.662(45) \mathrm{e}^{2} \mathrm{fm}^{2}$ for ${ }^{136} \mathrm{Xe}$, respectively. This enhancement for larger $\mathrm{N} / \mathrm{Z}$ is only partly observed in the results of the QPM calculation which also shows an increasing total strength but the dependency is much weaker. Also, for the more proton-rich nuclei much more strength is predicted by the QPM than observed experimentally.

Since the fragmentation of the strength is well reproduced by the QPM it is now possible to study the effect of the limited experimental sensitivity on this integral quantity. For the values given by the open squares $\left(\mathrm{QPM}_{L}\right)$ only the states above the experimental sensitivity limit of the corresponding experiments have been included in the total sum. The agreement between experiment and calculation is clearly improved by accounting

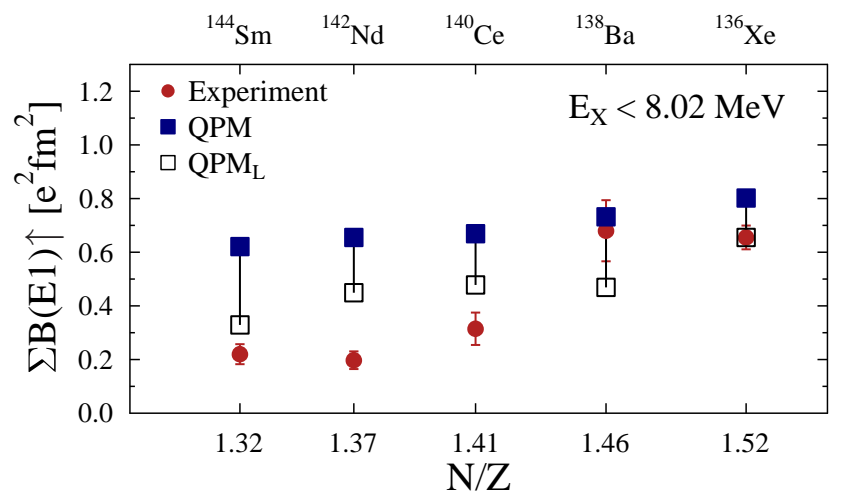

FIG. 8: (Color online) Integrated E1 strength for all stable even $\mathrm{N}=82$ isotones up to an excitation energy of $8.02 \mathrm{MeV}$ $\left(S_{n}\right.$ of $\left.{ }^{136} \mathrm{Xe}\right)$ as a function of neutron-to-proton ratio $\mathrm{N} / \mathrm{Z}$. For the QPM calculations two values are given, one including all states (QPM) and one including states above the corresponding experimental sensitivity limits only $\left(\mathrm{QPM}_{L}\right)$.

for the experimental sensitivity. Since the fragmentation increases for higher proton numbers (and thus smaller $\mathrm{N} / \mathrm{Z}$ ) the influence of the sensitivity limit becomes also more important in these nuclei. The experimentally observed strong enhancement of the total $\mathrm{B}(\mathrm{E} 1)$ strength for larger $\mathrm{N} / \mathrm{Z}$ ratio, thus, seems to be partly due to the higher fragmentation of the PDR in the more proton-rich nuclei and the consequently larger amount of experimentally unobserved strength. However, only part of the enhancement is due to this effect and also the full QPM calculation shows an enhancement of the total strength with increasing neutron-to-proton ratio. This comparison implies that the consideration of the experimental sensitivity limits plays an important role in the systematics of integral quantities.

\section{CONCLUSIONS}

We have measured the E1 strength distribution up to the neutron threshold in the semi-magic nucleus ${ }^{136} \mathrm{Xe}$ using the NRF method completing a systematic survey on the stable even $\mathrm{N}=82$ isotones to investigate the PDR. Similar as in the other $\mathrm{N}=82$ isotones, the $\mathrm{E} 1$ strength shows a resonance-like concentration well below the neutron separation energy which shows a strong fragmentation. A detailed comparison of the experimental results to calculations within the QPM has been performed for the investigated $\mathrm{N}=82$ isotones. The good agreement of the calculations in the fragmentation of the E1 strength confirms that the coupling of a collective mode to complex configurations used in the model is an accurate description of the damping of the PDR. Concerning the integrated strength, the influence of the limited experimental sensitivity has been investigated by applying the same limit to the QPM results. The agreement of calculation and experiment is improved by considering the ex- 
TABLE II: Summary of results on the systematics in the stable even $\mathrm{N}=82$ isotones. Energy values of the $2_{1}^{+}$and $3_{1}^{-}$states are taken from [43].

\begin{tabular}{lc|ccc|ccc}
\hline \hline Isotope & $\mathrm{N} / \mathrm{Z}$ & $\begin{array}{r}\mathrm{E}\left(2_{1}^{+}\right) \\
(\mathrm{MeV})\end{array}$ & $\begin{array}{c}\mathrm{E}\left(3_{1}^{-}\right) \\
(\mathrm{MeV})\end{array}$ & $\begin{array}{c}\mathrm{E}\left(1_{1}^{-}\right) \\
(\mathrm{MeV})\end{array}$ & \multicolumn{3}{|c}{$\begin{array}{c}\sum_{B(E 1) \uparrow}{ }^{a} \\
\left(\mathrm{e}^{2} \mathrm{fm}^{2}\right)\end{array}$} \\
\hline & & & & & $\mathrm{Exp}$. & $\mathrm{QPM}$ & $\mathrm{QPM}_{L}$ \\
\hline${ }^{136} \mathrm{Xe}$ & 1.518 & 1.313 & 3.275 & $(3.738)^{b}$ & $0.655(44)$ & 0.802 & 0.655 \\
${ }^{138} \mathrm{Ba}$ & 1.464 & 1.436 & 2.881 & 4.026 & $0.68(11)$ & 0.732 & 0.469 \\
${ }^{140} \mathrm{Ce}$ & 1.413 & 1.596 & 2.464 & 3.644 & $0.314(60)$ & 0.669 & 0.478 \\
${ }^{142} \mathrm{Nd}$ & 1.366 & 1.576 & 2.084 & 3.424 & $0.197(33)$ & 0.655 & 0.449 \\
${ }^{144} \mathrm{Sm}$ & 1.322 & 1.660 & 1.810 & 3.424 & $0.219(37)$ & 0.621 & 0.329 \\
\hline \hline
\end{tabular}

${ }^{a} \mathrm{up}$ to an excitation energy of $\mathrm{E}=8.06 \mathrm{MeV}\left(\mathrm{S}_{n}\right.$ of $\left.{ }^{136} \mathrm{Xe}\right)$.

${ }^{b}$ Unknown parity.

perimental sensitivity limit. This reveals that part of the experimentally observed enhancement of the integrated strength for larger neutron-to-proton ratio is due to the different influences of the experimental sensitivity limit due to a different amount of fragmentation along this chain of isotones. But even with this effect taken into account the enhancement of the strength within the PDR region with larger $\mathrm{N} / \mathrm{Z}$ ratio remains which fits into the picture of an oscillation of a neutron skin as the origin of the low-lying E1 strength.

In this paper, we have presented that the fragmentation of the PDR observed in high-resolution photon scattering experiments on the one hand provides in itself an observable to compare to theoretical model predictions to study the damping mechanism of this mode and on the other hand has an important impact on the experimental determination of integral quantities such as the total integrated strength. However, for a further investigation of the structure of the PDR, complementary experiments to photon scattering are mandatory. Recent experiments using the method of $\left(\alpha, \alpha^{\prime} \gamma\right)$ [44] on $\mathrm{Z}=50$ and $\mathrm{N}=82 \mathrm{nu}$ clei have revealed a splitting of the low-energy part of the E1 strength into two groups with different underly- ing structure 3, 37, 45]. These unexpected results show that especially experiments using complementary probes will provide additional observables to further constrain microscopic model calculations.

\section{Acknowledgments}

The authors thank the S-DALINAC group around R. Eichhorn for providing different beam set-ups and continous support during the measurements and acknowledge the help of M. Babilon, W. Bayer, D. Galaviz Redondo, T. Hartmann, B. Özel, and S. Volz during the beamtimes. We further thank P. von Brentano, U. Kneissl, M. Krticka, E. Litvinova, P. von Neumann-Cosel, A. Richter. A. Tonchev, J. Wambach, and H.J. Wörtche for stimulating discussions. This work was supported by the Deutsche Forschungsgemeinschaft (contracts SFB 634 and Zi 510/4-1) and by the Helmholtz Alliance Program of the Helmholtz Association, contract HA216/EMMI "Extremes of Density and Temperature: Cosmic Matter in the Laboratory".
[1] M. N. Harakeh and A. van der Woude, Giant Resonances (Oxford University Press, 2001).

[2] N. Paar, D. Vretenar, E. Khan, and G. Colo, Rep. Prog. Phys. 70, 691 (2007).

[3] J. Endres, E. Litvinova, D. Savran, P. A. Butler, M. N. Harakeh, S. Harissopulos, R.-D. Herzberg, R. Krücken, A. Lagoyannis, N. Pietralla, et al., Phys. Rev. Lett. 105, 212503 (2010).

[4] G. A. Bartholomew, E. D. Earl, A. J. Ferguson, J. W. Knowles, and M. A. Lone, Adv. Nucl. Phys. 7, 229 (1973).

[5] F. R. Metzger, Phys. Rev. C 18, 1603 (1978).

[6] F. R. Metzger, Phys. Rev. C 18, 2138 (1978).

[7] A. Jung, S. Lindenstruth, H. Schacht, B. Starck, R. Stock, C. Wesselborg, R.-D. Heil, U. Kneissl, J. Mar- graf, H. H. Pitz, et al., Nucl. Phys. A584, 103 (1995).

[8] R.-D. Herzberg, P. von Brentano, J. Eberth, J. Enders, R. Fischer, N. Huxel, T. Klemme, P. von Neumann-Cosel, N. Nicolay, N. Pietralla, et al., Phys. Lett. B 390, 49 (1997).

[9] K. Govaert, F. Bauwens, J. Bryssinck, D. De Frenne, E. Jacobs, W. Mondelaers, L. Govor, and V. Y. Ponomarev, Phys. Rev. C 57, 2229 (1998).

[10] N. Ryezayeva, T. Hartmann, Y. Kalmykov, H. Lenske, P. von Neumann-Cosel, V. Y. Ponomarev, A. Richter, A. Shevchenko, S. Volz, and J. Wambach, Phys. Rev. Lett. 89, 272502 (2002).

[11] A. Zilges, S. Volz, M. Babilon, T. Hartmann, P. Mohr, and K. Vogt, Phys. Lett. B 542, 43 (2002).

[12] J. Enders, P. von Brentano, J. Eberth, A. Fitzler, 
C. Fransen, R.-D. Herzberg, H. Kaiser, L. Käubler, P. von Neumann-Cosel, N. Pietralla, et al., Nucl. Phys. A724, 243 (2003).

[13] T. Hartmann, M. Babilon, S. Kamerdzhiev, E. Litvinova, D. Savran, S. Volz, and A. Zilges, Phys. Rev. Lett. 93, 192501 (2004).

[14] R. Schwengner, G. Rusev, N. Benouaret, R. Beyer, M. Erhard, E. Grosse, A. R. Junghans, J. Klug, K. Kosev, L. Kostov, et al., Phys. Rev. C 76, 034321 (2007).

[15] S. Volz, N. Tsoneva, M. Babilon, M. Elvers, J. Hasper, R.-D. Herzberg, H. Lenske, K. Lindenberg, D. Savran, and A. Zilges, Nucl. Phys. A779, 1 (2006).

[16] D. Savran, M. Fritzsche, J. Hasper, K. Lindenberg, S. Müller, V. Y. Ponomarev, K. Sonnabend, and A. Zilges, Phys. Rev. Lett. 100, 232501 (2008).

[17] A. P. Tonchev, S. L. Hammond, J. H. Kelley, E. Kwan, H. Lenske, G. Rusev, W. Tornow, and N. Tsoneva, Phys. Rev. Lett. 104, 072501 (2010).

[18] A. Makinaga, R. Schwengner, G. Rusev, F. Dönau, S. Frauendorf, D. Bemmerer, R. Beyer, P. Crespo, M. Erhard, A. R. Junghans, et al., Phys. Rev. C 82, 024314 (2010).

[19] G. Rusev, R. Schwengner, R. Beyer, M. Erhard, E. Grosse, A. R. Junghans, K. Kosev, C. Nair, K. D. Schilling, A. Wagner, et al., Phys. Rev. C 79, 061302 (2009).

[20] U. Kneissl, H. H. Pitz, and A. Zilges, Prog. Part. Nucl. Phys. 37, 349 (1996).

[21] T. Aumann, Eur. Phys. Journal A 26, 441 (2005).

[22] P. Adrich, A. Klimkiewicz, M. Fallot, K. Boretzky, T. Aumann, D. Cortina-Gil, U. Datta Pramanik, Th. W. Elze, H. Emling, H. Geissel, et al. (LAND-FRS Collaboration), Phys. Rev. Lett. 95, 132501 (2005).

[23] A. Klimkiewicz, N. Paar, P. Adrich, M. Fallot, K. Boretzky, T. Aumann, D. Cortina-Gil, U. D. Pramanik, T. W. Elze, H. Emling, et al. (LAND Collaboration), Phys. Rev. C 76, 051603 (2007).

[24] O. Wieland, A. Bracco, F. Camera, G. Benzoni, N. Blasi, S. Brambilla, F. C. L. Crespi, S. Leoni, B. Million, R. Nicolini, et al., Phys. Rev. Lett. 102, 092502 (2009), URL http://link.aps.org/abstract/PRL/v102/e092502,

[25] K. Sonnabend, D. Savran, J. Beller, M. A. Büssing, A. Constantinescu, M. Elvers, J. Endres, M. Fritzsche, J. Glorius, J. Hasper, et al., Nucl. Instr. and Meth. A 640, 6 (2011).

[26] M. Brunken, S. Döbert, R. Eichhorn, H. Genz, H.-D. Gräf, H. Loos, A. Richter, B. Schweizer, A. Stascheck, and T. Wesp, Nucl. Instr. and Meth. A 429, 21 (1999).

[27] W. Bayer, R. Eichhorn, M. Gopych, H.-D. Gräf, J. Hasper, A. Richter, and A. Zilges, Nucl. Instr. and Meth. A 575, 321 (2007).

[28] H. von Garrel, P. von Brentano, C. Fransen, G. Friess- ner, N. Hollmann, J. Jolie, F. Käppeler, L. Käubler, U. Kneissl, C. Kohstall, et al., Phys. Rev. C 73, 054315 (2006).

[29] R. Reifarth, M. Heil, F. Käppeler, F. Voss, K. Wisshak, F. Becvac, M. Kritcka, R. Gallino, and Y. Nagai, Phys. Rev. C 66, 064603 (2002).

[30] G. Rupp, D. Petrich, F. Käppeler, J. Kaltenbaek, B. Leugers, and R. Reifarth, Nucl. Instr. and Meth. A 608, 152 (2009).

[31] F. Ajzenberg-Selove, Nucl. Phys. A506, 1 (1990).

[32] S. Agostinelli, J. Allison, K. Amako, J. Apostolakis, H. Araujo, P. Arce, M. Asai, D. Axen, S. Banerjee, G. Barrand, et al., Nucl. Instr. and Meth. A 506, 250 (2003).

[33] J. Allison, K. Amako, J. Apostolakis, H. Araujo, P. A. Dubois, M. Asai, G. Barrand, R. Capra, S. Chauvie, R. Chytracek, et al., IEEE Trans. Nucl. Sci. 53, 270 (2006).

[34] N. Pietralla, Z. Berant, V. N. Litvinenko, S. Hartman, F. F. Mikhailov, I. V. Pinayev, G. Swift, M. W. Ahmed, J. H. Kelley, S. O. Nelson, et al., Phys. Rev. Lett. 88, 012502 (2002).

[35] M. A. Büssing, M. Elvers, J. Endres, J. Hasper, A. Zilges, M. Fritzsche, K. Lindenberg, S. Müller, D. Savran, and K. Sonnabend, Phys. Rev. C 78, 044309 (2008).

[36] W. Andrejtscheff, C. Kohstall, P. von Brentano, C. Fransen, U. Kneissl, N. Pietralla, and H. H. Pitz, Phys. Lett. B 506, 239 (2001).

[37] J. Endres, D. Savran, A. M. v. d. Berg, P. Dendooven, M. Fritzsche, M. N. Harakeh, J. Hasper, H. J. Wörtche, and A. Zilges, Phys. Rev. C 80, 034302 (2009).

[38] R. Schwengner, G. Rusev, N. Tsoneva, N. Benouaret, R. Beyer, M. Erhard, E. Grosse, A. R. Junghans, J. Klug, K. Kosev, et al., Phys. Rev. C 78, 064314 (2008).

[39] V. G. Soloviev, Theory of Atomic Nuclei: Quasiparticles and Phonons (Institute of Physics, Bristol, 1992).

[40] R.-D. Herzberg, C. Fransen, P. von Brentano, J. Eberth, J. Enders, A. Fitzler, L. Käubler, H. Kaiser, P. von Neumann-Cosel, N. Pietralla, et al., Phys. Rev. C 60, 051307 (1999).

[41] V. Yu. Ponomarev, V. G. Soloviev, Ch. Stoyanov, and A. I. Vdovin, Nucl. Phys. A323, 446 (1979).

[42] C. Bertulani and V. Yu. Ponomarev, Phys. Rep. 321, 139 (1999).

[43] ENSDF, NNDC Online Data Service, ENSDF database, http://www.nndc.bnl.gov/ensdf/.

[44] D. Savran, A. M. van den Berg, M. N. Harakeh, K. Ramspeck, H. J. Wörtche, and A. Zilges, Nucl. Instr. and Meth. A 564, 267 (2006).

[45] D. Savran, A. M. van den Berg, M. N. Harakeh, J. Hasper, A. Matic, H. J. Wörtche, and A. Zilges, Phys. Rev. Lett. 97, 172502 (2006). 